\title{
La importancia de la endoscopía dinámica en las alteraciones de vías aéreas altas en caballos de deporte
}

\section{The Relevance of Dynamic Endoscopy in Sport Horses Upper Airway Disseases}

\author{
Natalia Zimin Veselkoff \& Felipe Corrêa* \\ Universidad Andres Bello, Facultad de Recursos Naturales, Escuela de Medicina Veterinaria \\ * Autor de correspondencia: felipe2983@gmail.com
}

\section{RESUMEN}

Las enfermedades respiratorias de vías altas en los equinos son la segunda causa de pérdida deportiva después de las alteraciones músculo-esqueléticas, las cuales pueden generar intolerancia al ejercicio que se evidencia a través de una disminución del rendimiento deportivo. Los trastornos obstructivos de las vías respiratorias altas (VRA) se producen durante el ejercicio del caballo siendo un problema común entre los caballos de carrera, afectando a un $70 \%$ de los pacientes con historial de ruido respiratorio o bajo rendimiento. La evaluación de las vías respiratorias altas del equino se realiza por endoscopía. Sin embargo muchas alteraciones de las VRA son de naturaleza dinámica, las cuales se producen solo durante el ejercicio, por lo que no son necesariamente evidentes durante un exámen endoscópico en reposo. Por lo cual, la cinta de alta velocidad (treadmill) y los estudios de endoscopía dinámica en terreno, han revelado que el colapso dinámico de las VRA es una importante causa de los malos resultados en caballos de deporte. Existen variadas formas de colapso dinámico de las VRA, que se han reportado y en muchos casos hasta aproximadamente el $50 \%$ son múltiples estructuras colapsando simultáneamente. En Chile no existe el diagnóstico mediante endoscopía dinámica lo que limita un correcto diagnóstico por endoscopía en reposo, por lo que puede resultar en fracasos terapéuticos que se traducen en pérdidas económicas.

Palabras claves: caballo; endoscopía dinámica; colapso dinámico.

\section{SUMMARY}

Upper aiway disseases in horses are the second leading cause of performance loss after musculoskeletal pathologies, which can cause exercise intolerance that is evidenced by a decrease in athletic performance. Obstructive disorders of upper respiratory tract (URT) are produced during horse's exercise, and it's a common problem among racehorses, affecting $70 \%$ of patients with a respiratory noise or poor performance. The evaluation of horse's upper airway is performed by an URT endoscopy. However many alterations of equine's URT are dynamic in nature, which are produced just during exercise, so they are not necessarily evident during an endoscopic examination at rest. Therefore, the treadmill (high-speed tape) and dynamic endoscopy studies in the field have revealed that the dynamic collapse of the URT is a major cause of poor performance in sport horses. In Chile there is no dynamic endoscopy availiable, which limits the correct diagnosis and can result in treatment failure and economic losses.

Keywords: horse; dynamic endoscopy; dynamic collapse. 


\section{INTRODUCCIÓN}

Las enfermedades respiratorias en los equinos generan un deterioro en el intercambio gaseoso y como consecuencia, intolerancia al ejercicio evidenciado a través de una disminución del rendimiento deportivo (McCann, 2000; Davidson et al., 2011; Franklin et al., 2012). En el caballo, existen obstrucciones dinámicas del sistema respiratorio superior, las cuales se producen cuando no se pueden tolerar los cambios de presión durante el ejercicio debido a que estas estructuras no tienen soporte óseo (Go et al., 2014a; Franklin y Allen 2015). Estos trastornos obstructivos de las vías respiratorias altas (VRA) son un problema común entre los caballos de carrera, afectando a un $70 \%$ de los pacientes con historial de ruido respiratorio y/o bajo rendimiento, resultando en la segunda causa de la pérdida del rendimiento deportivo (Franklin et al., 2010; Barakzai y Cheetham 2012; Stand et al., 2012).

Durante el ejercicio intenso se generan dramáticos aumentos en el flujo de aire, junto con cambios en las presiones de las VRA (Franklin y Allen 2015). El caballo es un respirador nasal obligado, por lo que las obstrucciones dinámicas son considerablemente mayores en esta especie en comparación a otras, por lo que es limitante en su rendimiento (Franklin y Allen 2015).

De acuerdo a Stand (2012), las obstrucciones en las VRA a menudo generan ruidos respiratorios y es motivo de preocupación para el jinete, lo que puede disminuir el valor comercial del animal. Estas pueden ser causadas por múltiples condiciones, las cuales son diagnosticas mediante endoscopía en reposo, siendo algunas indetectables o mal diagnosticadas (Stand et al., 2012; Mirazo et al., 2014).

El examen endoscópico en caballos de deporte se realiza de forma rutinaria para la evaluación de las VRA y se ha convertido en una herramienta importante del examen de pre-compra. Este examen se utiliza para ayudar en la evaluación del caballo y su futuro deportivo (Leuton y Lumsdem 2013). Muchas alteraciones de las VRA del equino son de naturaleza dinámica, por lo que no son necesariamente evidentes durante un examen en reposo, por lo cual, el treadmill y los estudios de endoscopía dinámica en terreno, han revelado que el colapso dinámico de las VRA es una importante causa de los malos resultados en caballos de deporte (Van Erck-Westergren, et al., 2013).

Se describe que en el 50\% de los caballos con alteraciones de las VRA no se evidencian anormalidades durante un examen endoscópico en reposo y la obstrucción sólo se convierte evidente durante el ejercicio (Franklin y Allen 2015). Por lo tanto, en el diagnóstico de las alteraciones de VRA, la videoendoscopía dinámica juega un rol fundamental y es considerada como "Gold Standard" para hacer un diagnóstico definitivo del colapso dinámico en las VRA en los caballos, mientras que los hallazgos en reposo, frecuentemente son poco fiables o inexistentes (Barakzai y Cheetham 2012; Van Erck-Westergren et al., 2013).

Entre las afecciones más comunes de las VRA se encuentran; neuropatía laríngea recurrente, desviación axial de los pliegues ariepiglóticos, colapso de las cuerdas vocales, desplazamiento dorsal intermitente del paladar blando entre otras (Pollock et al., 2013; Mirazo et al., 2014; Franklin y Allen, 2015). El hecho de que algunas de estas alteraciones sean transitorias y autolimitantes complica aún más el panorama para los Médicos Veterinarios que tratan de proporcionar terapias efectivas para caballos afectados por la inestabilidad dinámica de la faringe o laringe (Pollock et al., 2013).

Existen variadas formas de colapso dinámico de las VRA, que se han informado y en muchos casos hasta aproximadamente el 50\% son múltiples estructuras colapsando simultáneamente (Mirazo et al., 2014; Franklin et al., 2015). Los signos clínicos suelen ser inespecíficos, subclínicos o sólo aparentes bajo esfuerzo máximo, lo que plantea un desafío diagnóstico bajo condiciones normales de descanso (Davidson et al., 2011; Hawkins, 2015). La verdadera prevalencia de los trastornos en las VRA es difícil de determinar, por la naturaleza dinámica de estas, el Médico Veterinario debe considerar la importancia funcional de los hallazgos endoscópicos para cada atleta (Franklin y Allen 2015).

A pesar de los recientes avances en el diagnóstico de las VRA, los pacientes sin hallazgos durante la endoscopía en reposo, se someten a cirugía, basada únicamente en la descripción del entrenador, propietario o de un ruido anormal (Stand et al., 2012; Barnett et al., 2013). Las razones para esto son la falta de disponibilidad de equipos de diagnóstico y las limitaciones financieras (Stand et al., 2012). 


\section{GENERALIDADES ANATÓMICAS Y FUNCIONALES DEL SISTEMA RESPIRATORIO ALTO EN EQUINOS}

La función principal del sistema respiratorio es entregar oxígeno y eliminar el dióxido de carbono de la sangre (McGrorum 2007). El aire es transportado hacia los pulmones a través de diversas estructuras de las vías respiratorias hasta llegar a los alvéolos, en donde se lleva a cabo el intercambio gaseoso (McGrorum 2007; Couêtil y Hawkins 2013).

Las vías respiratorias están conformadas por las fosas nasales, vías nasales rostrales, tabique nasal, cornetes, senos paranasales, bolsas guturales, nasofaringe, paladar duro, paladar blando, faringe, laringe, tráquea, bronquios, bronquiolos y alveolos (Couêtil y Hawkins 2013).

El caballo es un respirador nasal obligado, por lo tanto las VRA sufren grandes cambios de presión y de flujo de aire durante el ejercicio, estas generan una gran resistencia al flujo de aire y pueden ser un factor limitante en la capacidad de ejercicio del caballo (Hinchcliff et al., 2008; Ducharme 2012; Couêtil y Hawkins 2013). Los cambios en la resistencia durante la inspiración y la espiración pueden explicarse por los cambios de presión en las VRA (Hinchcliff y col, 2008).

Estas estructuras se encuentran soportadas en algunas zonas por hueso (Cavidad nasal) o cartílago (Laringe, tráquea y bronquios) y en otras zonas sólo por músculo (Ollares y faringe) (Couêtil y Hawkins 2013). El hecho de que algunas porciones no están soportadas por estructuras rígidas las hace más susceptibles al colapso durante el ejercicio intenso (Hinchcliff et al., 2008; Ducharme 2012).

\section{ANATOMÍA Y FUNCIÓN DE LA FARINGE}

La faringe es un saco músculo-membranoso en forma de embudo que representa tanto una porción del tracto digestivo así como del tracto respiratorio. La faringe conecta la cavidad nasal a la laringe (Rush y Mair 2004; Hinchcliff et al., 2008). Es más amplio y más grande de rostral a caudal. La faringe está constituida por los músculos palatino y pterigoideo, huesos hioides (Rush y Mair 2004; Ducharme 2012). La cavidad de la faringe tiene siete aberturas: Las dos coanas (posterior a las narinas), dos aperturas faríngeas de las trompas auditivas (Trompas de Eustaquio), apertura oral, apertura laríngea y apertura del esófago (Rush y Mair 2004; Hinchcliff et al., 2008).

El paladar blando se extiende caudalmente desde el paladar duro a la base de la laringe, tiene una longitud de aproximadamente $13 \mathrm{~cm}$ de largo y este separa la nasofaringe de la orofaringe (Allen 2015). Presenta una membrana mucosa oral sobre su superficie ventral, y una mucosa respiratoria en su superficie dorsal. En el fondo del paladar blando se encuentran las glándulas palatinas, la aponeurosis palatina, y los músculos palatinos y palatofaríngeos (Rush y Mair 2004).

La posición del velo del paladar está determinada por la actividad de diferentes grupos de músculos antagonistas, incluyendo el músculo elevador del velo palatino, el músculo tensor del velo palatino, palatinos y palatofaríngeos (Rush y Mair 2004; Hinchcliff et al., 2008; Ducharme, 2012). El músculo elevador del velo palatino eleva el paladar blando durante la deglución y la vocalización. El músculo tensor del velo palatino amplía la nasofaringe durante la inspiración tensando la aponeurosis palatina, deprimiendo la parte rostral del paladar blando hacia la lengua (Rush y Mair, 2004; Ducharme, 2012).

Los músculos palatinos y palatofaríngeos controlan la posición de la mitad caudal del paladar blando. La contracción de los músculos palatinos y palatofaríngeos acorta el paladar blando y deprime la parte caudal hacia la lengua.

La inervación del paladar blando es a través de la rama faríngea del nervio vago, rama mandibular del nervio trigémino y el nervio glosofaríngeo. El margen libre caudal del paladar blando continúa dorsalmente a ambos lados de la laringe para formar el "ostium bucale" (Arco palatofaríngeo) (Rush y Mair 2004; Hinchcliff et al., 2008; Ducharme 2012; Allen 2015). 
La laringe es un conducto entre la faringe y la tráquea que contribuye a las funciones de la respiración, vocalización, y deglución (Auer y Stick 2011). Se compone de cuatro estructuras cartilaginosas: epiglotis, tiroides, cricoides y dos cartílagos aritenoides. El cartílago tiroides articula con el cartílago cricoides a través de la articulación cricotiroidea (Ducharme 2012). El ligamento cricotiroideo se extiende por el cartílago tiroides y cricoides en la cara ventral de la laringe. El espacio cricotraqueal es la unión entre el cartílago cricoides y la tráquea (Couêtil y Hawkins 2013). El cartílago cricoides se articula con el aritenoides, esta articulación es importante porque permite los movimientos de los cartílagos aritenoides. Los aritenoides poseen un proceso corniculado, vocal, y muscular, siendo que entre estos últimos se encuentra el cuerpo del aritenoide (Auer y Stick 2011).

\section{EVALUACIÓN ENDOSCÓPICA}

La endoscopía del aparato respiratorio es el método diagnóstico de elección para las enfermedades del VRA de los equinos (Mirazo et al., 2014). La mayoría de los diagnósticos se pueden realizar a partir de un examen completo en reposo (McGorum 2007). Sin embargo existen estudios que han documentado que algunos trastornos son subestimados o que no pueden ser diagnosticados durante un examen endoscópico en reposo por la naturaleza dinámica de las patologías (Van Erck 2011; Parente et al., 2012; Mirazo et al., 2014; Joo, et al., 2015). El desarrollo de la endoscopía dinámica permitió evaluar patologías dinámicas de las VRA para la detección de alteraciones en nasofaringe y laringe durante el ejercicio del caballo, lo que ha facilitado la detección de condiciones que no son evidentes durante el examen en reposo (Barakzai y Cheetham 2012; Kumas y Maden. 2013; Mirazo et al., 2014;).

\section{EFECTOS DE LOS SEDANTES EN LA EVALUACIÓN ENDOSCÓPICA}

La sedación puede alterar la función laríngea, en un grado que los diagnósticos resultan en falsos positivos, ya que provoca una acentuación de paresia, que se puede interpretar como hemiparesia (Slovis 2004). Por lo tanto el paciente no debe ser sedado durante la endoscopía y sólo debe ser contenido con restricción física, para asegurar que no interfiera la sedación con la evaluación de la vía aérea (Rush y Mair 2004; Parente et al., 2012).

\section{ENDOSCOPÍA EN REPOSO}

La endoscopía en reposo se realiza de forma rutinaria en el paciente de pie usando restricción física (Slovis 2004). La técnica consiste en la introducción del endoscopio por uno de los ollares, atravesando fosas nasales, dirigiéndolo hacia el meato ventral, mientras la curvatura del endoscopio se dirige hacia dorsal para observar la apertura del seno maxilar y luego avanzada para observar el cornete etmoidal (Slovis 2004; Parente et al., 2012). El endoscopio se posiciona hacia la zona ventrocaudal de la faringe, en donde se observa la nasofaringe, paladar blando, aperturas de las bolsas guturales, el receso faríngeo dorsal y la porción superior de la laringe (Slovis 2004; Parente et al., 2012; Couêtil y Hawkins 2013). 


\section{ENDOSCOPÍA DINÁMICA}

La evaluación endoscópica dinámica de la laringe y faringe durante el ejercicio se ha utilizado para identificar diversas condiciones de las VRA que no se pueden identificar en un examen endoscópico en reposo (Hewson y Arroyo 2015). La endoscopía dinámica puede llevarse a cabo en treadmill o en terreno (Franklin y Allen 2015). Aunque la evaluación mediante treadmill puede identificar condiciones dinámicas, esta técnica no puede replicar las condiciones en terreno, la adherencia y las condiciones ambientales que se pueden alterar en el examen (Mirazo et al., 2014), además de la intervención del jinete, como el uso de espuelas, el cambio de la marcha y curvas cerradas, las cuales afectan a la inestabilidad de la VRA (Taralyn et al., 2015), por lo tanto desde el punto de vista clínico, un factor clave, es la capacidad de replicar el motivo de consulta durante la prueba de esfuerzo (Franklin y Allen 2015). La endoscopía dinámica en terreno permite que el caballo sea montado en su entorno normal, a diferencia de treadmill, que no permite la simulación en la superficie (Taralyn et al., 2015).

De acuerdo a McCarrel y Woodie (2015) el 50\% de los caballos que presenta una evaluación sin anormalidades en reposo, tendrán una obstrucción dinámica en una evaluación durante el ejercicio, y de estos el 19\% a 56\% de los caballos tendrán anomalías múltiples (Strand y Skjerve 2012; Mirazo et al., 2014; McCarrel y Woodie 2015). Kelly (2013) describe que la forma de posicionar el endoscopio, es de igual forma que la endoscopía en reposo, a diferencia de esta el endoscopio queda fijo (Kelly et al., 2013). La forma de ajustar el endoscopio, es colocarlo justo rostral a la epiglotis para permitir la visualización clara de la nasofaringe caudal y la laringe (McGorum et al., 2007).

En un examen endoscópico dinámico aparentemente normal en caballos, se debe observar una abducción máxima de los cartílagos aritenoides durante el ejercicio, la mayor parte del proceso corniculado debe estar horizontalmente a $90^{\circ}$ respecto a la línea media de la hendidura glótica y no tiene que existir movimiento de los cartílagos aritenoides (Rush y Mair 2004; Compostella et al., 2014).

El examen endoscópico durante el ejercicio es considerado "Gold Standard" para evaluar con precisión las causas dinámicas de obstrucción de las VRA, entregando un diagnóstico definitivo (Witte $e t$ al., 2011; Van Erck-Westergren et al., 2013) y además permite evidenciar la eficacia del tratamiento médico o quirúrgico. No obstante, como resultado de la práctica y de las consideraciones económicas, esto es rara vez posible en la práctica clínica (McGorum 2007; Mirazo et al., 2014).

\section{INFLUENCIA DE LA POSICIÓN DE LA CABEZA Y CUELLO EN LAS ALTERACIONES DINÁMICAS DE LAS VRA}

Las obstrucciones dinámicas de las VRA se producen cuando no se puede soportar los cambios de presión durante el ejercicio debido a que estas estructuras no tienen soporte óseo por lo que son propensas al colapso (Go et al., 2014a). De acuerdo a esto, diversos estudios han demostrado que un cambio en el diámetro de la faringe depende de la posición de la cabeza y cuello (Van Erck- Westergren et al., 2013; Go et al., 2014b; Zebisch et al., 2014).

Se describe un efecto significativo en la disminución del diámetro en la faringe cuando se flexiona la cabeza y el cuello, esto hace que disminuya el flujo inspiratorio de aire y se colapsen las VRA (Van Erck-Westergren et al., 2013; Zebisch et al., 2014). Estudios concordaron que el aumento de la flexión de la cabeza y cuello incide en alteraciones de las VRA como el desplazamiento dorsal del paladar blando (Berkley y Whitfield-Cargile 2015; Joo, et al., 2015).

El trastorno se caracteriza por colapso simétrico bilateral de las cuerdas vocales y cartílagos aritenoides, y es inducida por el cambio en la posición de la cabeza que se produce cuando los jinetes frenan durante el ejercicio (Fjordbakk et al., 2012; Zebisch et al., 2014). Los caballos afectados tienen un aspecto endoscópico normal de la laringe en reposo y también cuando se evalúa con endoscopía en ejercicio con la cabeza libre de movimientos, pero al momento de flexionar la cabeza y cuello, se ve alterado el diámetro de las VRA (Fjordbakk et al., 2012; Zebisch et al., 2014).

Se ha considerado un factor predisponente la posición de la cabeza y el cuello para el colapso de las VRA, pero dependiendo de la actividad que se haga, se verán más o menos afectados. En el caso de los 
caballos de carrera, estos corren con el cuello extendido y por lo tanto no se ven afectados, pero cuando los frenan en las primeras etapas de la carrera, estos se verán afectados disminuyendo su rendimiento (Strand et al., 2012; Berkley y Whitfield-Cargile 2015; Franklin y Allen 2015).

\section{RUIDOS RESPIRATORIOS}

Las consecuencias más frecuentes de las obstrucción de las VRA son el pobre rendimiento y el ruido respiratorio anormal, siendo esto último la presentación clínica más común para las alteraciones de estas vías (Witte et al., 2011; Franklin y Allen 2015).

De acuerdo a un estudio en el cual demostraron mediante grabaciones digitales, utilizando micrófonos, que los sonidos respiratorios ayudarían en el diagnóstico diferencial, dependiendo de su naturaleza, si es que los ruidos son de origen inspiratorios o espiratorios (Witte et al., 2011).

Allen y Franklin (2010) mencionan que las anormalidades de las VRA son muy probables que se observen en caballos que hacían un ruido anormal durante el entrenamiento y menos probables en los que no hacían ruidos anormales en ejercicio (Allen y Franklin 2010, 2013). Por esto la endoscopía dinámica está indicada para los caballos que presentan ruido respiratorio durante el ejercicio (Hewson y Arroyo 2015).

A pesar de los recientes avances en el diagnóstico de las obstrucciones dinámicas de las VRA, algunos caballos sin hallazgos durante la endoscopía en reposo se someten a cirugía de las vías respiratorias basada únicamente en la descripción del entrenador del propietario o del ruido anormal y como resultando en fracasos terapéuticos (Witte et al., 2011).

\section{OBSTRUCCIONES DINÁMICAS DE LAS VÍAS RESPIRATORIAS ALTAS EN EL EQUINO}

Varios estudios concordaron que las patologías más frecuentes de caballos sometidos a endoscopía dinámica con antecedentes de ruidos respiratorios y/o intolerancia al ejercicio fueron las siguientes: desviación axial de los pliegues ariepigloticos (11\% a 40\%), el colapso de las cuerdas vocales (7\% a $41 \%)$, neuropatía laríngea recurrente $(8 \%$ a $38 \%$ ) y el desplazamiento dorsal del paladar blando (16\% a $40 \%)$. Las cuales se presentaron como patologías múltiple (24\% a $57 \%$ de los equinos) o únicas (Barakzai y Dixon 2011; Van Erck et al., 2011; Witte et al., 2011; Barakzai y Cheetham 2012; Strand et al., 2012; Mirazo et al., 2014).

Neuropatía laríngea recurrente:

La neuropatía laríngea recurrente (NLR o hemiplejia laríngea idiopática) ha sido identificada como una causa común de malos resultados para los caballos que compiten en una variedad de disciplinas deportivas (Barnett et al., 2013; Franklin y Allen 2015; Hawkins 2015). Esta condición ha sido estudiada respecto a la función respiratoria, ya que tiene un impacto significativo sobre esta durante el ejercicio (Collins et al., 2009; Barakzai y Cheetham 2012; Van Erck- Westergren et al., 2013).

Se ha sugerido que esta condición surge debido a una axonopatía distal degenerativa progresiva, que afectan a los nervios laríngeos recurrentes (Compostella et al., 2014; Hawkins 2015). Cursa con una progresiva parálisis muscular, producto de una desmielinización de los axones del nervio laríngeo recurrente (Witte et al., 2011; Hawkins 2015) que inervan los músculos aductores de la laringe (Cricoaritenoideo lateral, vocal, ventricular, aritenoideo transverso y el músculo abductor cricoaritenoideo dorsal) (Garrett et al., 2011).

Esta parálisis muscular conduce a una atrofia neurogénica de la musculatura intrínseca de la laringe, sobre todo el músculo cricoaritenoideo dorsal (Couêtil y Hawkins 2013). Como resultado de la inervación anormal, el colapso dinámico del aritenoides en la vía aérea interfiere con la función respiratoria y conduce a la intolerancia al ejercicio y el ruido respiratorio anormal (Hawkins, 2015). Esta parálisis puede conducir a un colapso parcial o total de los cartílagos aritenoides (Barakzai y Cheetham 2012) siendo la disfunción del lado izquierdo la más común. 
Otras posibles causas de colapso aritenoideo incluyen la ingestión de neurotoxinas, micosis de las bolsas guturales, abscesos o tumores de la cabeza y el cuello, o daño iatrogénico al nervio laríngeo recurrente como consecuencia de un trauma o de inyección peri vascular de la vena yugular, en las cuales se verán afectados ambos cartílagos aritenoides (Franklin y Allen 2015).

Se describen como factores de riesgo a los caballos de razas de mayor tamaño, por lo que este vínculo entre el tamaño corporal y NLR sugiere que debiese haber una crianza selectiva para reducir NLR (McGorum et al., 2007; Boyko et al., 2014). Además se vieron afectados factores genéticos en la heredabilidad de NLR en los cuales se identificaron dos regiones genómicas con evidencias sugerentes para la asociación de los cromosomas 21 y 31 (Dupuis et al., 2013).

Como prevalencia la mayor población de razas estudiada relacionada con NRL, es la pura sangre Ingles, donde se reporta que entre el 2,6 a $8 \%$ de los caballos son afectados por la NLR (Witte et al., 2011).

Los signos clínicos se producen por la disfunción del músculo CAD, que resulta en un mayor o menor grado la función de los cartílagos aritenoides que se ve afectada, el cual conduce a un aumento de la resistencia inspiratoria (McGorum et al., 2007; Barakzai y Cheetham 2012; Cramp y Barakzai 2012; Hawkins 2015).

Existen varios sistemas para la clasificación de la función laríngea en reposo, de los cuales los sistemas de 4 grados (Havemeyer) y 5 grados (Lane), se han correlacionado con la función laríngea durante el ejercicio, mediante endoscopía dinámica en numerosos estudios (Barakzai y Dixon 2011; Froydenlund y Dixon 2014; Chesen y Whitfield-Cargile 2015).

El diagnóstico de la NLR se hace por endoscopía dinámica o en reposo, dependiendo del grado de parálisis del cartílago aritenoides de la laringe, donde se evalúan el movimiento de los cartílagos aritenoides y la simetría de la hendidura glótica (Lindegaard et al., 2007).

La función laríngea puede ser evaluada a través de la endoscopía en reposo, sin embargo la endoscopía durante el ejercicio se justifica en los casos que exista una función laríngea deficiente de los cartílagos aritenoides la cual no puede ser pesquisada mediante endoscopía en reposo (Van ErckWestergren et al., 2013), debido a que el grado asignado durante la evaluación en reposo no predice necesariamente el grado de disfunción de laringe y obstrucción durante el ejercicio extenuante (Compostella et al., 2014).

Más recientemente, el uso de la ultrasonografía laríngea ha sido descrito como una herramienta complementaria para el diagnóstico de NLR (Van Erck- Westergren et al., 2013; Hawkins, 2015). Esta técnica ha demostrado ser extremadamente precisa en la predicción de la disfunción del aritenoides durante el ejercicio, con una sensibilidad de $90 \%$ y especificidad del $98 \%$ en comparación con la endoscopía de reposo (Sensibilidad del 80\% y especificidad del 81\%) (Garrett et al., 2011). Por lo tanto, la ecografía de laringe es considerado un método de diagnóstico útil para la evaluación de la función del cartílago aritenoides en caballos con los hallazgos en reposo erróneos, especialmente donde no está disponible la endoscopía dinámica (Van Erck- Westergren et al., 2013; Hawkins 2015).

El objetivo del tratamiento para NLR es doble: Estabilizar la vía aérea y restaurar la mecánica del flujo de aire a la normalidad y para reducir el ruido respiratorio asociado con el flujo de aire anormal (McGorum et al., 2007). Un sin número de tratamientos quirúrgicos se han descrito para la NLR, siendo el de elección la técnica quirúrgica "Tie-back", en la cual se sustituye la función del músculo cricoaritenoideo dorsal mediante el anclaje del cartílago aritenoides al tiroides y así mantenerlo en abducción permanente (Cramp y Barakzai 2012; Van Erck- Westergren et al., 2013; Rossignol et al., 2014). Las tasas de éxito para "Tie-back" están en alrededor del $90 \%$ para los caballos que realizan ejercicio sub máximo, sin embargo las tasas de éxito de los caballos de carreras son sustancialmente más bajos, que van desde alrededor de 60 de 78\% (Van Erck Westergren et al., 2013).

Desplazamiento dorsal intermitente del paladar blando:

Se describe que el desplazamiento dorsal intermitente del paladar blando (DDIPB) como "La condición que ocurre durante el ejercicio, cuando el borde caudal del paladar blando queda desplazado a 
una posición por encima de la epiglotis, lo que resulta en la obstrucción de la rima glotis" (Allen 2015; Franklin y Allen 2015; Mirazo et al., 2015). En circunstancias normales, el paladar blando siempre debe permanecer en una posición subepiglotica, excepto durante la deglución (Allen 2015).

En un estudio se identificó que los caballos con DDIPB podrían tener una relación diferente entre la laringe y el aparato hioideo, denominada conformación laringohioidea (Parente et al., 2012). Es este, se midió la profundidad del cuerpo del hueso hioides en la base del proceso lingual, la ubicación basihioides fue significativamente más superficial (ventral) en los caballos afectados con DDIPB en comparación con los caballos no afectados (Parente et al., 2012). Además, está asociado a la presencia DDIPB, la hipoplasia, flacidez o deformidad de la epiglotis, evidenciando en la exploración endoscópica, la hipoplasia epiglotica en la cual en un equino adulto se observaría corta y estrecha (Auer y Stick 2011).

El aumento de la flexión de cabeza y cuello aumenta la incidencia de DDIPB por una combinación en el aumento de las presiones negativas de las vías aéreas y la retracción caudal de la laringe (Berkley y Whitfield-Cargile 2015; Franklin y Allen 2015).

Los caballos con DDIPB suelen tener un historial de rendimiento pobre, intolerancia al ejercicio y ruidos respiratorios además de detenerse durante la carrera (Allen 2015). Barakzai y Hawkes (2010) describen que en un 70 a $80 \%$ de los casos, los malos resultados se asocian con un ruido respiratorio espiratorio, descrito por los entrenadores o jinetes como "ronquidos" (Barakzai y Hawkes 2010; Couêtil y Hawkins 2015). No obstante el DDIPB afecta tanto a la inspiración y expiración, pero este último generalmente es en mayor medida (Offord et al., 2014). Sin embargo, en un estudio se identificó que el sonido no fue detectado consistentemente en todos los caballos, un 30\% de los caballos son conocidos por ser DDIPB "silenciosos" (Barakzai y Hawkes 2010).

Se conoce como factores de riesgo el grupo de edad más común afectado con DDIPB entre 2-3 años de edad caballos fina sangre de carrera y standartbred, ya que el uso de riendas y la posición de la cabeza y cuello relacionada con estos deportes influyen en la presentación del DDPB (Couêtil y Hawkins 2013). Se ha reportado que DDIPB que se produce en el 1,3\% de los caballos en general, pero se estima que existe una mayor prevalencia en los caballos fina sangre de carreras (Berkley y Whitfield-Cargile 2015).

Para el diagnóstico de DDIPB se realiza a través de endoscopía, ya sea en reposo en el caso que sea un DDPB persistente o durante el ejercicio. En general, la observación de DDIPB en reposo es un examen predictivo positivo bastante confiable, pero en resultados negativos el examen es muy deficiente para descartar la presencia de DDISP (Couêtil y Hawkins 2013; Allen 2015; Berkley y Whitfield-Cargile 2015).

Es de suma importancia realizar un examen endoscópico en reposo de la nasofaringe para descartar causas físicas de la disfunción del paladar como quistes faríngeos, atrapamiento epiglótico o parálisis faríngea (Barakzai y Hawkes 2010; Allen 2015). DDPB e inestabilidad palatina (IP) son condiciones dinámicas, y los hallazgos de la endoscopía en reposo se ha demostrado que se correlacionan deficientemente con los hallazgos endoscópicos dinámicos (Barakzai y Hawkes 2010; Allen 2015).

Un estudio mostró que la obtención de un diagnóstico utilizando la historia y la endoscopía en reposo resultó en una tasa de diagnóstico erróneo del 35\% (Allen 2015). Es por esto que la evaluación mediante endoscopía dinámica es el método diagnostico Gold Standard demostrando que DDIPB ocurre con mayor frecuencia durante o al final del ejercicio extenuante (Allen 2015; Berkley y Whitfield-Cargile 2015). Esta condición se diagnostica si es que el paladar permanece dorsal a la epiglotis durante más de 8 segundos (Barakzai y Hawkes 2010; Couêtil y Hawkins 2013; Allen 2015).

Existe una fuerte evidencia de que la posición de la cabeza y el cuello influye en el DDIPB, por lo tanto la endoscopía en ejercicio debe similar las condiciones normales, la posición de la cabeza y cuello durante la actividad que realiza el paciente, para obtener un diagnóstico preciso (Couêtil y Hawkins 2013; Berkley y Whitfield-Cargile 2015; Taralyn et al., 2015).

Más recientemente en un estudio se informó que el DDIPB se observó con mayor frecuencia en los caballos examinados mediante endoscopía dinámica evaluados por Treadmill que los que fueron evaluados mediante endoscopía dinámica en terreno, ya que en la cinta de alta velocidad, pueden llegar a correr distancias más largas y por ende demostrar DDIPB (Offord et al., 2014; Allen, 2015). 
Otra técnica diagnóstica es la ecografía la cual tiene un alto valor predictivo negativo (89\%), pero un valor predictivo positivo bajo (36\%), lo que significa se puede descartar con certeza razonable usando ultrasonido de laringe, pero eso se debe tener cuidado al hacer un diagnóstico positivo de DDIPB utilizando este método, en comparación a la endoscopía en reposo (Barakzai y Hawkes 2010).

El tratamiento conservador debe llevarse a cabo inicialmente para todos los casos de DDIPB los que no responden a este, deben ser considerados para el tratamiento quirúrgico (Couêtil y Hawkins, 2013).

La posición de la lengua ha sido discutida como una posible causa de DDPB y por esto se discute el tratamiento conservador que consiste en el amarre de lengua, en el cual se ancla la lengua a la mandíbula impidiendo que el aparato hioideo se mueva hacia craneal (Barakzai y Hawkes 2010; Berkley y Whitfield-Cargile 2015) pero no existe ninguna evidencia científica sólida para su uso (Barakzai y Hawkes 2010; Berkley y Whitfield-Cargile 2015).

El collar de Cornell es un dispositivo externo que soporta la laringe durante el ejercicio (Couêtil y Hawkins 2013, Allen 2015). El collar tiene un mecanismo de elevación termoplástico, que se mueve la laringe en una posición más rostral y dorsal imitando la acción del músculo tirohioideo (Couêtil y Hawkins 2013). El uso de este collar debe considerar el manejo del caballo inicialmente diagnosticado con DDIPB. Los caballos que no responden al tratamiento conservador deben ser considerados para el tratamiento quirúrgico (Couêtil y Hawkins 2013; Allen 2015).

El método preferido de tratamiento quirúrgico es el Tie-Forward (Couêtil y Hawkins 2013; Offord et al., 2014; Allen 2015) Esta técnica consiste colocar suturas o prótesis entre el cartílago tiroides de la laringe y el cuerpo del hueso hioides intentando recrear la acción del músculo tirohioideo (Offord et al., 2014). El procedimiento Tie-Forward ha demostrado radiográficamente al

mover la laringe hacia rostral y dorsal mientras que el cuerpo del hueso hioides se vuelve a colocar en sentido caudal y dorsal (Offord et al., 2014; Allen 2015). Esta técnica presenta un rango de éxito entre 66\%-81\% descrito por Berkley y Whitfield-Cargile (2015) (Berkley y Whitfield-Cargile 2015).

El rendimiento del paciente se ve afectado por esta condición, en la cual los entrenadores están dispuestos a probar intervenciones conservadoras y quirúrgicas. Sin embargo las tasas de éxito son limitadas por lo tanto se espera que exista una mejor comprensión de la etiopatogenia de DDPB, la cual permitirá el desarrollo de tratamientos más eficaces en el futuro (Allen 2015).

La medición más precisa del éxito antes de la intervención quirúrgica y después de ella, es el examen endoscópico dinámico, para luego obtener una evaluación significativa (Barakzai y Hawkes 2010). Sin embargo el diagnóstico requiere endoscopía dinámica, la cual no está ampliamente disponible (Chalmers et al., 2009).

Desviación axial de los pliegues ariepigloticos:

La desviación axial de los pliegues ariepigloticos se produce durante la inspiración (Slovis 2004; Couêtil y Hawkins 2013). Los pliegues ariepiglóticos generalmente se mantienen tensos entre el cartílago aritenoides y la epiglotis, al contrario de la desviación axial de los pliegues ariepigloticos (DAPA) en la cual existe pérdida de la abducción de estos, disminuyendo la tensión (McGroum 2007). Sin embargo, el grado de desviación puede ser leve, moderada o severo (Slovi 2004).

Desviación axial del pliegues ariepiglóticos generalmente precede el desplazamiento dorsal del paladar blando y a la disfunción palatina (Slovis 2004; Franklin y Allen 2010). Esto podría estar relacionado con el aumento de la presión inspiratoria que obliga a los pliegues ariepiglóticos desviarse axialmente y luego el desplazamiento dorsal del paladar blando (Slovis 2004). Este colapso dinámico de los pliegues ariepiglóticos se produce durante el ejercicio de alta velocidad (Couêtil y Hawkins 2013). La etiología de DAPA es idiopática, los investigadores han especulado que podría ser una disfunción neurológica o de una patogenia similar a la del desplazamiento dorsal del paladar blando (Couêtil y Hawkins 2013). Además se describe que el movimiento y posición de la cabeza y cuello influyen la presencia del DAPA (Terron-Canedo et al., 2011; Franklin y Allen 2015).

Normalmente el caballo presenta ruidos inspiratorios durante el ejercicio máximo (Couêtil y Hawkins 2013; Hawkins 2015) además de intolerancia al ejercicio. El trastorno generalmente suele ser 
confundido con DDPB y NLR (Couêtil y Hawkins 2013). Suelen ser más afectados caballos jóvenes, menores a 3 años y la presencia de alteraciones de las VRA como el desplazamiento dorsal del paladar blando (Hawkins 2015). En una investigación en Sudáfrica se detectó que en un $40 \%$ de los caballos de carrera con signología respiratoria manifestó DAPA, presentándose únicamente o en conjunto con otras anomalías dinámicas (Mirazo et al., 2014). Por otra parte se registró la presencia de DAPA como la segunda causa de colapso dinámico con una prevalencia del 34\% (Barnett et al., 2013).

El diagnóstico de esta alteración dinámica no se puede hacer mediante endoscopía en reposo por lo tanto el diagnóstico correcto es a través de endoscopía dinámica ya sea Treadmill o durante el ejercicio en terreno bajo esfuerzo máximo (Auer y Stick 2011; Mirazo et al., 2014; Hawkins 2015). La condición generalmente afecta a ambos pliegues ariepigloticos, sin embargo se han visto afectado unilateralmente (Auer y Stick 2011). DAPA en algunos caballos puede progresar a través de todos los grados, dependiendo del nivel de fatiga o solo puede cursar con un grado de desviación (Slovis 2004).

Se han descrito varias alternativas de tratamiento para DAPA, en los cuales la resección de los pliegues ariepigloticos, se indica cuando existe evidencia de la desviación de los pliegues ariepigloticos y para esto es necesario el diagnostico mediante endoscopía dinámica (Hawkins 2015; Auer y Stick 2015). El tratamiento quirúrgico se puede realizar por resección del pliegue ariepiglótico afectado, realizado mediante cirugía por distintos abordajes tales como por transendoscópicas, utilizando laser con el paciente de pie (Auer y Stick 2011; Hawkins 2015) o se puede realizar a través de laringotomía (Terron-Canedo 2011). Aunque los caballos jóvenes también pueden ser tratados con antiinflamatorios obteniendo un éxito moderado, los rendimientos de resección quirúrgica son aun mayor y requiere un período más corto de tiempo de reposo (Hawkins 2015).

Se indica que el pronóstico después de la resección quirúrgica de la pliegues ariepiglóticos es bueno para el desarrollo deportivo del paciente (Couetil y Hawkins 2013).

Colapso de las cuerdas vocales:

El colapso de las cuerdas vocales $(\mathrm{CCV})$ surge por la pérdida de la función del músculo CAD generando una disminución de la tensión de las cuerdas vocales, predisponiéndolo a colapsar durante el ejercicio (Munroe y Weese 2011; Hawkins 2015) La pérdida de la abducción del cartílago aritenoides reducirá la tensión en la cuerda vocal (Munroe y Weese 2011). El CCV ocurre secundariamente al colapso del cartílago aritenoides (Hawkins 2015). Experimentalmente se ha demostrado que podría ocurrir CCV con posterioridad a una disfunción del CAD (Franklin y Allen 2015), estando asociado con la neuropatía laríngea recurrente (NLR) el cual tendrá efectos sobre las características mecánicas de las cuerdas vocales (Barakzai y Dixon 2011). Sin embargo no está claro si CCV representa una etapa temprana o una manifestación diferente de la NLR (Franklin y Allen 2015).

Colapso dinámico de uno o ambos pliegues vocales se produce generalmente durante la inspiración, el cual es causante de ruidos respiratorios que en este caso corresponde a un "silbido" (Munroe y Weese 2011). Se observa con mayor frecuencia en conjunto con el colapso del cartílago aritenoides, además de una baja en el rendimiento (McGorum 2007; Franklin y Allen 2015). En algunos equinos, se observa el CCV y en otros la dilatación de ventrículo detrás de la cuerda vocal (Auer y Stick 2011; Franklin y Allen 2015). Está condición se asocia a la posición de la cabeza y cuello con la presencia del CCV y la presencia de neuropatía laríngea recurrente (McGorum 2007; Munroe y Weese 2011; Fjordbakk et al., 2013; Franklin y Allen 2015).

De acuerdo a Mirazo (2014), el CCV se ha detectado en 35\% de los pacientes que presentaron ruidos respiratorios y/o disminución del rendimiento, el cual se puede encontrar como la única anomalía de las VRA, o puede ser asociado con NLR (Mirazo et al., 2014). Por otra parte Barakzai y Dixon (2011) describen que el $\mathrm{CCV}$ era un hallazgo frecuente $(23,1 \%)$ en poblaciones de caballos sometidos a endoscopía dinámica. Dada su naturaleza dinámica, el CCV sólo puede ser diagnosticado mediante endoscopía en ejercicio (Auer y Stick 2011). El tratamiento de elección para el CCV es la ventriculocordectomia mediante laringotomía, la resección de ambas cuerdas no es recomendada ya que esto puede generar un exceso de tejido cicatricial obstruyendo la rima glotis (Munroe y Weese 2011). 
El pronóstico para el paciente que presenta $\mathrm{CCV}$, con tratamiento quirúrgico mediante la ventriculocordectomia para el ruido respiratorio es bueno, no obstante para mejorar en el rendimiento deportivo es reservado (Munroe y Weese 2011).

\section{CONCLUSIONES}

Se podría concluir que los beneficios expuestos por diversos autores acerca de la endoscopía dinámica son que resulta fundamental para tener un diagnóstico certero, y así realizar un correcto tratamiento. Hay patologías que se pueden diagnosticas por endoscopía en reposo, pero el 50\% de los caballos que tengan un examen endoscópico normal, tendrán alguna o múltiples alteraciones si es que se realiza una evaluación con endoscopía durante el ejercicio, por lo tanto esto genera un reto en el diagnóstico para los Médicos veterinarios que no puedan realizar la endoscopía dinámica (Mirazo et al., 2014; Franklin et al., 2010; Franklin y Allen 2015; McCarrel y Woodie 2015).

Existen alteraciones de las VRA que solo pueden ser diagnosticadas por endoscopía dinámica, tales como la neuropatía laríngea recurrente (Grado III.1 y III.2), desplazamiento dorsal intermitente del paladar blando, desviación axial de los pliegues ariepigloticos, colapso de las cuerdas vocales, colapso faríngeo y laríngeo, entre otras (Parente et al., 2012; Stand et al., 2012; Barakzai y Cheetham 2012; Franklin y Allen 2015; Allen 2015; Berkley y Whitfield-Cargile 2015).

Varios estudios concordaron con que las alteraciones dinámicas más frecuentes en los equinos fueron; neuropatía recurrente laríngea, desplazamiento dorsal intermitente del paladar blando, desviación axial de los pliegues ariepigloticos y el colapso de las cuerdas vocales. Pero estas no siempre serán únicas, existen varios pacientes que presentan un conjunto de alteraciones, alrededor del $50 \%$ de los caballos que presenten alteración en las VRA, serán múltiples estructuras colapsando, lo que hace indispensables, diagnosticar mediante video endoscopía (Mirazo et al., 2014; Franklin y Allen 2015). El examen endoscópico dinámico, no siempre es sensible ya que existen condiciones que no son las mismas a las de la carrera o disciplina deportiva que realice el caballo, como la intensidad del ejercicio, superficie de la pista, posición de la cabeza y cuello, jinete, variables ambientales, entre otras, por lo que es deficiente en algunos sentidos. Sin embargo es la mejor herramienta diagnostica para alteraciones de las vías respiratorias altas (Barakzai y Cheetham 2012; Van Erck-Westergren et al., 2013; Zebisch et al., 2014; Go et al., 2014 b; Joo, et al., 2015; Hewson y Arroyo 2015).

En Chile no existe el diagnóstico por endoscopía dinámica por lo cual no tenemos un diagnóstico definitivo para las alteraciones de las VRA durante el ejercicio, por lo tanto se ve bastante limitado el correcto diagnóstico por endoscopía en reposo. Sin embargo otras técnicas diagnosticas como el ultrasonido han dado buenos resultados en donde debemos sacar provecho de este examen complementario.

\section{REFERENCIAS}

Allen, K.J. y S.H Franklin. 2010. Assessment of the exercise tests used during overground endoscopy in UK Thoroughbred racehorses and how these may affect the diagnosis of dynamic upper respiratory tract obstructions. Equine Veterinary Journal. Supplement, 38, 587-591.

Allen, K.J. y S.H. Franklin. 2013. Characteristics of palatal instability in Thoroughbred racehorses and their association with the development of dorsal displacement of the soft palate. Equine Veterinary Journal 45, 454-459.

Allen, K.J. 2015. Soft palate displacement in horses. In Practice September 37, 415-421.

Auer, J.A. y J.A Stick. 2011. Equine Surgery W.B. Saunders Company 4 edicion, 45, pp 592- 620. 
Sustainability Agri, Food and Environmental Research 4(3), 2016: 1-15 ISSN: 0719-3726

Barakzai, S. Z. y J. Cheetham. 2012. Endoscopic examination of exercising horses: effects on diagnosis and treatment of upper respiratory tract disorders. Equine Veterinary Journal, 44,501-503.

Barakzai, S. Z y P.M Dixon. 2011. Correlation of resting and exercising endoscopic findings for horses with dynamic laryngeal collapse and palatal dysfunction. Equine Veterinary Journal, 43(1), 18-23.

Barakzai, S. Z. y C.S Hawkes. 2010. Dorsal displacement of the soft palate and palatal instability. Equine Veterinary Education, 22, 253-264.

Barnett, T. P, J.M O'Leary, T.H. Parkin, P.M Dixon, y S.Z Barakzai. 2013. Long-term exercising videoendoscopic examination of the upper airway following laryngoplasty surgery: a prospective crosssectional study of 41 horses. Equine Veterinary Journal, 45, 593-597.

Berkley, A. y C. Whitfield-Cargile. 2015. Update on Diseases and Treatment of the Pharynx Vet Clin Equine, 31 1-11.

Boyko, A. R, S.A Brooks, A. Behan-Braman, M. Castelhano, E. Corey, K.C Oliveira y N.E Robinson. 2014. Genomic analysis establishes correlation between growth and laryngeal neuropathy in Thoroughbreds. BMC Genomics, 15(1), 1-17.

Chalmers, H. J, A.E Yeager y N. Ducharme. 2009. Ultrasonographic assessment of laryngohyoid position as a predictor of dorsal displacement of the soft palate in horses. 42 Veterinary Radiology \& Ultrasound the Official Journal Of The American College Of Veterinary Radiology And The International Veterinary Radiology Association, (1).

Chesen, A. B, y C. Whitfield-Cargile. 2015. Update on Diseases and Treatment of the Pharynx. Veterinary Clinics Of North America: Equine Practice, 31 (Respiratory Medicine and Surgery), 1-11.

Compostella, F. W.H. Tremaine y S.H. Franklin. 2014. Retrospective study investigating causes of abnormal respiratory noise in horses following prosthetic laryngoplasty. Equine Veterinary Journal. Supplement 43, 27-30.

Couêtil, L. y J. Hawkins. 2013. Respiratory diseases on the horse. Manson Publishing, UK. pp 926, 164-168.

Collins, N. E. Milne. C. Hahn y P. Dixon. 2009. Correlation of the Havemeyer endoscopic laryngeal grading system with histopathological changes in equine cricoarytenoideus dorsalis muscles. Irish Veterinary Journal, 62, 334-338.

Cramp, P. y S.Z. Barakzai. 2012. Surgical management of recurrent laryngeal neuropathy. Equine Veterinary Education, 24, 307-321.

Davidson, E.J. M. Harris, B. Benson, B.B Martin, R. Nolen-Walston, R.C. Boston, y V. Reef. 2011. Exercising blood gas analysis, dynamic upper respiratory tract obstruction, and postexercising bronchoalveolar lavage cytologyda comparative study in poor performing horses. Journal of Equine Veterinary Science 31, 475-480.

Ducharme, N.G. 2012. Equine Surgery. Sciencie direct, Ebscohost, Chapter 44, pp 569-591.

Dupuis, M. Z. Zhang, K. Durkin, C. Charlier, P. Lekeux, y M. Georges. 2013. Detection of copy number variants in the horse genome and examination of their association with recurrent laryngeal neuropathy. Animal Genetics, (2). 
Sustainability Agri, Food and Environmental Research 4(3), 2016: 1-15 ISSN: 0719-3726

Franklin, S.H. y J.K Allen. 2015 Assessment of dynamic upper respiratory tract function in the equine. Equine Veterinary Education. 1-12.

Franklin, S.H. S.H Barakzai, P. Couroucé-Makblanc, P. Dixon, KJ. Nankervis, J.D Perkins, A. Roberts, E. Vanerk-Westergren, y J. Allen. 2010. Investigation of the incidence and type of injuries associated with high-speed treadmill exercise testing. Equine Veterinary Journal, 38 70-75.

Franklin, S. H. E. Van Erck-Westergren, y W.M Bayly. 2012. Respiratory responses to exercise in the horse. Equine Veterinary Journal, 44(6), 726-732.

Froydenlund, T.J. y P.M Dixon. 2014. A review of equine laryngoplasty complications. Equine Veterinary Education, 26, 98-106.

Fjordbakk, C.T. H.J Chalmers, S.J Holcombe, y E. Strand. 2013. Results of upper airway radiography and ultrasonography predict dynamic laryngeal collapse in affected horses. Equine Veterinary Journal 45, 705-710.

Garrett, K.S. J.B Woodie, y M. Embertson. 2011. Association of treadmill upper airway endoscopic evaluation with results of ultrasonography and resting upper airway endoscopic evaluation. Equine Veterinary Journal 43, 365-371.

Go, L. A.K Barton, y B. Ohnesorge. 2014a. Objective classification of different head and neck positions and their influence on the radiographic pharyngeal diameter in sport horses. BMC Veterinary Research. Doi: 10.1186/1746-6148-10-118.

Go, L. A.K Barton, y B. Ohnesorge. 2014b. Pharingeal diameterr in various head and neck positions during exercise in sport horses. BMC Veterinary Research. Doi: 10.1186/176-6148- 10-117.

Hawkins, J. 2015. Advances in Equine Respiratory Surgery Wiley Blackwell, Indiana, USA, Wiley Blackwell, pp 60-80.

Hewson, J. y L.G Arroyo. 2015. Respiratory disease diagnostic spproaches in the horse. Vet Clin Equine. Doi: 0.1016/j.cveq.2015.04.008.

Hinchcliff, K. R.J Geor, y A.J Kaneps. 2008. Equine Exercice Physiology Saunders Elsevier Capitulo 3.1 pp 172-176.

Joo, K., O. Szenci, Z. Bohak, A. Povazsa, y O. Kutasia. 2015. Evaluation of Overground Endoscopy Findings in Sport and Pleasure Horses. Journal of Equine Veterinary Science 1-7.

Kelly, P. G. R.M Reardon, M.S Johnston, y P. Pollock. 2013. Comparison of dynamic and resting endoscopy of the upper portion of the respiratory tract in 57 Thoroughbred yearlings. Equine Veterinary Journal, 45(6), 700-704.

Kumas, C. y M. Maden. 2013. Evaluation of The Dynamic (Overground) Endoscopy Procedure in The Diagnosis of Upper Respiratory Tract Diseases Affecting Performance of Racehorses. Kafkas Universitesi Veteriner Fakultesi Dergisi, A55-A60.

Leuton, J.L y L. Lumsdem. 2013. Dynamic respiratory endoscopic pre and post laringoplasty in Thoroughbred horses racehorse. Equine Veterinary Journal 45, 700-704. 
Sustainability Agri, Food and Environmental Research 4(3), 2016: 1-15 ISSN: 0719-3726

Lindegaard, C. L. Husted, H. Ullum, y H. Fjeldborg. 2007. Sedation with detomidine and acepromazine influences the endoscopic evaluation of laryngeal function in horses. Equine Veterinary Journal 36, 553556.

McCann, J. 2000. Differential diagnosis of abnormal respiratory noises in the exercising horse http://inpractice.bmj.com (Acceso, 23 agosto, 2015) 22: 370-381.

McCarrel, T.M. y B.J Woodi. 2015. Update on Laryngeal Disorders and Treatment. Vet Clin Equine 31, $13-26$.

McGorum, B.C. P.M. Dixon, E.N Robinson y J. Schumacher. 2007. Equine Medicine and Surgery Elsevier, Philadelphia, PA USA pp 3-13.

Mirazo, J. E. P. Page, L. Rubio-Martinez, H.J Marais, y Lyle. 2014. Dynamic upper respiratory abnormalities in Thoroughbred racehorses in South Africa. Journal Of The South African Veterinary Association, 85, 1-6.

Munroe, G.A. y J.S Weese. 2011. Equine clinical medicine, surgery and reproduction. Taylor y Francis Group. London, Manson. Pp 435-436.

Offord, S. L.K Tulloch, S.H Franklin, W.H Tremaine, N.S Woodford, y K.J Allen. 2014. The effect of the laryngeal tie-forward procedure and soft palate cautery on nasopharyngeal diameter in horses . Veterinary Record 1 of 4 doi: 10.1136/vr.102509.

Parente, E. J. S.H Franklin, F.J Derksen, M.A Weishaupt, H.J Chalmers, y C. Tessier. 2012. Chapter 42: Diagnostic Techniques in Equine Upper Respiratory Tract Disease. Equine Surgery, 536-557.

Pollock, P. J. P.G Kelly, R.M Reardon, y G.M Kelly. 2013. Dynamic ventrorostral displacement of the dorsal laryngeal mucosa in horses. The Veterinary Record 19, 501.

Rossignol, F. A. Vitte, J. Boening, M. Maher, A. Lechartier, O. Brandenberger, M.M Flores, L. Lang, W. Walker, y N.G Ducharme. 2014. Laryngoplasty in Standing Horses. Veterinary Surgery 9999, 1 - 7.

Rush. B y T. Mair. 2004. Equine Respiratory Disease. Publishing Blackwell Science. Oxford UK pp 8182.

Stand, E. C.T Fjordbakk, K. Sundberg, L. Spangen, H. Lunden, y S. Hanchen-Olsen. 2012. Relative prevalence of upper respiratory tract obstructive disorders in two breeds of harness racehorse (185 cases; 1998-2006) Equine Veterinary Journal 44, 518-523.

Strand, E., y E. Skjerve. 2012. Complex dynamic upper airway collapse: associations between abnormalities in 99 harness racehorses with one or more dynamic disorders. Equine Veterinary Journal 44, 524-528.

Slovis, N.M. 2004. Atlas of Equine Endoscopy, Mosby Westline Industrial Drive. St. Louis, Missouri pp 50-58.

Terrón-Canedo, N. y S. Franklin. 2013. Dynamic epiglottic retroversion as a cause of abnormal inspiratory noise in six adult horses. Equine Veterinary Education, 25(11), 565-56.

Taralyn. M. J. McCarrel, y B. Woodie. 2015. Update on Laryngeal Disorders and Treatment. Vet Clin Equine 31 13-26. 
Van Erck, E. 2011. Dynamic respiratory videoendoscopy in ridden sport horses: effect of head flexion, riding and airway inflammation in 129 cases. Equine Veterinary Journal. Supplement, 40, 18-24.

Van Erck-Westergren, E, S.H Franklin, y W.M Bayly. 2013. Respiratory diseases and their effects on respiratory function and exercise capacity. Equine Veterinary Journal 45, 376-387.

Witte, S.H.P. F.Harriss, G. Kelly y P. Pollock. 2011. Association of owner-reported noise with findings during dynamic respiratory endoscopy in Thoroughbred racehorses. Equine Veterinary Journal. 43, 9-17.

Zebisch, A. A. May, S. Reese, y H. Gehlen. 2014. Effects of different head-neck positions on the larynges of ridden horses. Journal of Animal Physiology \& Animal Nutrition, 98(5), 894- 900. 